\title{
Lipid-related protein NECTIN2 is an important marker in the progression of carotid atherosclerosis: An intersection of clinical and basic studies
}

\author{
Shen $\mathrm{Li}^{1,2}$, Yuan Gao${ }^{1}, \mathrm{Ke} \mathrm{Ma}^{3}$, Yanan $\mathrm{Li}^{1,2}$, Chen liu${ }^{1,2}$, Yingxue Yan ${ }^{1,2}$, Weishi Liu ${ }^{1,2}$, \\ Hongbing Liu ${ }^{1}$, Zhen $\mathrm{Li}^{3}$, Bo Song ${ }^{1}$, Yuming $\mathrm{Xu}^{1}$, Zongping $\mathrm{Xia}^{1,2}$ \\ 'Department of Neurology, the First Affiliated Hospital of Zhengzhou University, Zhengzhou 450052, Henan \\ Province, China; \\ ${ }^{2}$ Department of Clinical Systems Biology Laboratories, the First Affiliated Hospital of Zhengzhou University, \\ Zhengzhou 450052, Henan Province, China; \\ ${ }^{3}$ Department of Vascular Surgery, the First Affiliated Hospital of Zhengzhou University, Zhengzhou 450052 , \\ Henan Province, China.
}

Address for Correspondence: Dr. Zongping Xia, Department of Neurology and Department of Clinical Systems Biology Laboratories, the First Affiliated Hospital of Zhengzhou University, Zhengzhou 450052, Henan Province, China.

E-mail: zxia2018@zzu.edu.cn

Dr. Yuming Xu, Department of Neurology,

the First Affiliated Hospital of Zhengzhou

University, Zhengzhou 450052, Henan

Province, Chin

E-mail: xuyuming@zzu.edu.cn

\begin{tabular}{|l|}
\hline Access this article online \\
\hline Website: \\
www.intern-med.com \\
DOI: \\
10.2478/jtim-2021-0044 \\
\hline Quick Response Code: \\
\hline
\end{tabular}

\section{ABSTRACT}

Background: The nectin cell adhesion molecule 2 (NECTIN2) protein is a cell adhesion molecule involved in lipid metabolism. We aimed to explore the potential role of NECTIN2 in carotid atherosclerosis (CA). Materials and Methods: Patients who underwent carotid endarterectomy (CEA) at the First Affiliated Hospital of Zhengzhou University were enrolled in this study. APOE-- rats fed western or normal diet were used to model early pathological changes in CA. The relationship between patients' lipid indices and plaque severity was assessed using ordinal regression analysis. Mendelian randomisation (MR) analysis was used to determine the causal links between low-density lipoprotein cholesterol (LDL-C) and atherosclerosis. After matching analysis of the single-cell transcriptome and microarray data of carotid plaques, NECTIN2 was identified as a key factor affecting CA. The importance of NECTIN2 was further verified by immunofluorescence staining of CEA and APOE-1 rat specimens. Results: A total of 108 patients were included. The traditional lipid indices did not correlate significantly with the plaque severity $(P>0.05)$. NECTIN2 provided a strong causal link between LDL-C level and CA (MR effect size $>0$ ). Deep-sequencing data illustrated that NECTIN2 expression was cell specific. In early-stage CA, NECTIN2 expression was increased in endothelial cells; however, in advanced-stage CA, NECTIN2 was overexpressed in macrophages located in fibrous caps. APOE ${ }^{-/}$rat carotid artery and human carotid plaques modelled the entire atherosclerotic process, showing an upregulation of NECTIN2 expression in CA. Conclusions: Lipid-related protein NECTIN2 is a potential marker in CA progression and can potentially be a new therapeutic target for clinical prevention.

Key words: atherosclerosis, plaque, thrombectomy, NECTIN2, Mendelian randomisation, deep sequencing

\section{INTRODUCTION}

Carotid atherosclerosis (CA) can cause cerebrovascular diseases. ${ }^{[1]}$ The global prevalence of carotid plaque is $21.15 \%$ in people aged 30-79 years, affecting approximately 816 million people. ${ }^{[2]}$ The CA pathogenesis involves early intimal lipid deposition, intimal thickening, carotid artery stenosis and occlusion. ${ }^{[3,4]}$ The mechanism of CA is complicated, including lipid infiltration theory, inflammation theory, injury responsae theory, oxidative stress theory and so on. ${ }^{[5,6]}$

Lipid metabolism is vital in CA progression. ${ }^{[7]}$ The nectin cell adhesion molecule 2 (NECTIN2), a gene involved in atherosclerotic artery walls, is significantly downregulated in response to plasma cholesterol reduction, ${ }^{[8]}$ likely playing an important role in intercellular junction 


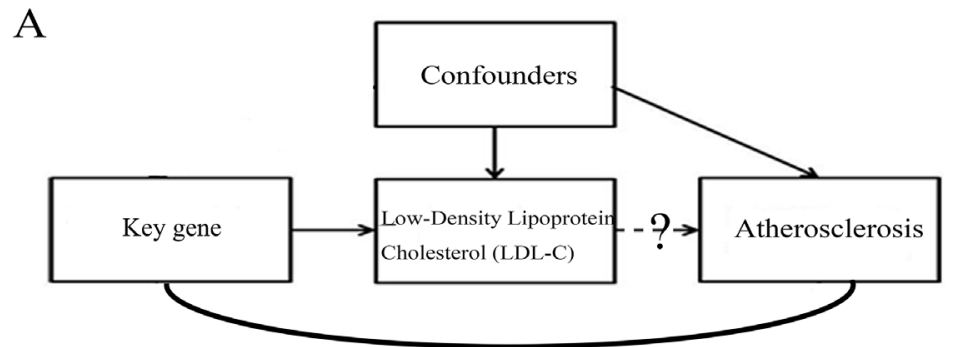

B
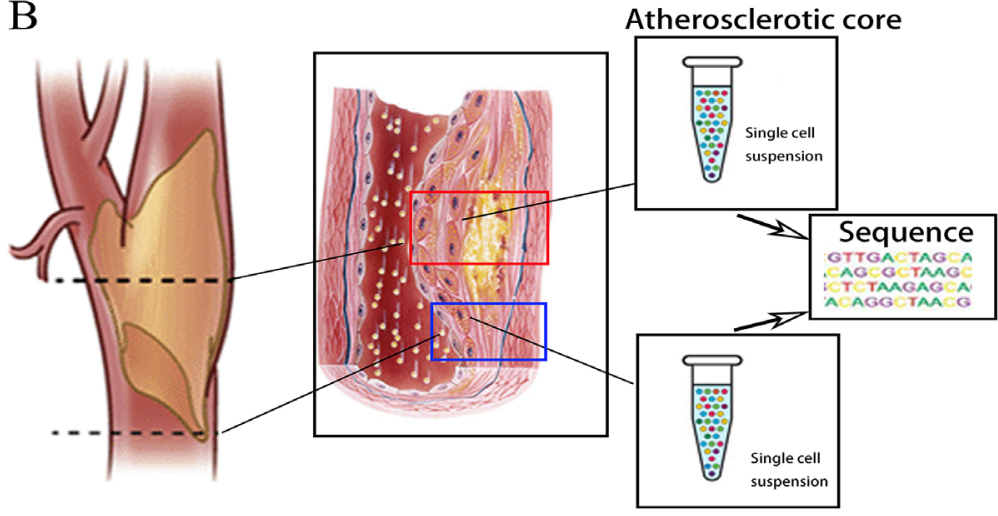

C

Proximal adjacent

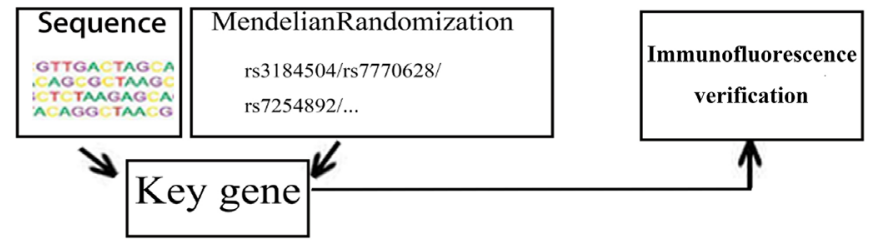

Figure 1: The overall route of the study. (A) Using Mendelian randomisation to study the causal link between LDL-C and carotid atherosclerosis, and revealing important single-nucleotide polymorphisms. (B) The differences between atherosclerotic core and proximal adjacent regions were found by single-cell RNA sequence technology. (C) The key genes related to carotid atherosclerosis are obtained by data matching and verified by models. LDL-C: low-density lipoprotein cholesterol.

assembly and inflammatory signalling pathways. ${ }^{[0,10]} \mathrm{A}$ recent study demonstrated that NECTIN2 gene might casually link to low-density lipoprotein cholesterol (LDL-C), which was analysed in the blood from 12,449 individuals.${ }^{[1]}$ However, there are no studies about the roles of NECTIN2 in CA progression.

Plaque specimens from carotid endarterectomy (CEA) are important in CA research. ${ }^{[12,13]}$ When fed western diet, $\mathrm{APOE}^{-/}$rats can model atherosclerotic events well and it is convenient to obtain specimens in early-stage CA. ${ }^{[14]}$ Through a combination of specimens from CEA, we can understand the entire CA progression better. Mendelian randomisation (MR) analysis can be used to find the genetic links between exposure and outcome (Figure 1A). ${ }^{[15]}$ Microarray and single-cell RNA sequencing (scRNAseq) technologies enable us to achieve high-throughput detection and transcriptomic analysis, ${ }^{[16,17]}$ which can help us understand the precise cell composition of biological samples..$^{[18]}$
In our study, we used MR studies to analyse singlenucleotide polymorphisms (SNPs) to explore the potential roles of NECTIN2 in CA. Also, deep mining of scRNAseq and microarray data was used to reveal correlation of NECTIN2 at the transcription level to CA progression. Then, we verified our results using $\mathrm{CEA}$ and $\mathrm{APOE}^{-/-}$rat specimens. Together, we aimed to analyse comprehensively about the potential roles of NECTIN2 in CA and provide a potential target for the clinical prevention of CA in the future.

\section{MATERIALS AND METHODS}

\section{Human carotid plaques and APOE-/- rat models}

Patients who underwent CEA at the First Affiliated Hospital of Zhengzhou University from January 2020 to December 2020 were included in this prospective cohort study. The inclusion criteria were as follows: (1) meeting the indication of CEA operation; ${ }^{[19]}(2)$ availability of complete results of colour ultrasound of carotid plaques (severity 
Li et al.: Lipid-related protein NECTIN2 is an important marker in the progression of carotid atherosclerosis

\begin{tabular}{|c|c|c|c|c|}
\hline \multirow[t]{2}{*}{ Variable } & \multicolumn{4}{|c|}{ Degree of carotid stenosis } \\
\hline & II & III & IV & $P$ \\
\hline Age (years, mean \pm SD) & $66.36 \pm 7.51$ & $65.45 \pm 6.99$ & $64.08 \pm 9.05$ & 0.32 \\
\hline Gender $(n)$ & 11 & 22 & 75 & 0.94 \\
\hline Male & 9 & 17 & 59 & \\
\hline Female & 2 & 5 & 16 & \\
\hline $\mathrm{TG}(\mathrm{mmol} / \mathrm{L})$ & $1.41 \pm 0.80$ & $1.43 \pm 0.83$ & $1.58 \pm 0.95$ & 0.39 \\
\hline $\mathrm{TC}(\mathrm{mmol} / \mathrm{L})$ & $3.96 \pm 1.03$ & $3.69 \pm 1.17$ & $3.72 \pm 0.98$ & 0.69 \\
\hline LDL-C (mmol/L) & $2.40 \pm 0.93$ & $2.22 \pm 0.94$ & $2.19 \pm 0.86$ & 0.39 \\
\hline $\mathrm{HDL}-\mathrm{C}(\mathrm{mmol} / \mathrm{L})$ & $1.13 \pm 0.39$ & $1.05 \pm 0.27$ & $1.05 \pm 0.26$ & 0.42 \\
\hline APOA $1(\mathrm{~g} / \mathrm{L})$ & $1.22 \pm 0.22$ & $1.30 \pm 0.39$ & $1.20 \pm 0.28$ & 0.39 \\
\hline APOB $(g / L)$ & $0.92 \pm 0.27$ & $0.78 \pm 0.30$ & $0.85 \pm 0.31$ & 0.65 \\
\hline
\end{tabular}

The degree of carotid stenosis was II (stenosis $<50 \%$ ), III (stenosis $\geq 50 \%$ and $<70 \%$ ) and IV (stenosis $\geq 70 \%$ ).

CEA: carotid endarterectomy; TG: triglyceride; TC: total cholesterol; LDL-C: low-density lipoprotein cholesterol; HDL-C: high-density lipoprotein cholesterol; APOA1: apolipoprotein A1; APOB: apolipoprotein B.

of the carotid plaques evaluated by the degree of carotid stenosis; Table 1); (3) blood samples tested within $12 \mathrm{~h}$ of admission (normal values of related indices are shown in Supplementary Table 1); (4) patients with complete clinical data and (5) age $>18$ years. Near full-thickness sections of arteries and plaques from the atherosclerotic core (AC) and full-thickness proximally adjacent (PA) arterial sections were recovered from patients (Figure 1B). The specimens were quickly frozen in liquid nitrogen and stored at $-80^{\circ} \mathrm{C}$ until use. To model the changes in early-stage CA, $12 \mathrm{APOE}^{-/}$rats were randomly divided into two groups based on their diets: western diet (WD) or normal diet (ND) (differential feeding from 7 to 18 weeks of age). All Sprague Dawley rats were male, and were maintained and cared for in accordance with the National Institutes of Health guidelines under a controlled environment $\left(21^{\circ} \mathrm{C} \pm\right.$ $2^{\circ} \mathrm{C}, 50 \% \pm 10 \%$ relative humidity and a 12 -h light and 12 -h dark cycle with lights on at $0700 \mathrm{~h}$ EST). Every week during the whole WD feeding period, blood samples were drawn from the tail veins, and the carotid arteries were checked using animal colour ultrasound to clearly monitor the occurrence of atherosclerotic plaques. At 5 and 11 weeks of the WD feeding period, carotid artery specimens were obtained. Our study was approved by the Ethics Committee of the First Affiliated Hospital of Zhengzhou University (2020-KY-0067-001), and all animal studies were approved by the Ethics Committee of the Institutional Animal Care and Use Committee at Zhengzhou University (2020-zzuIACUC-0172).

Carotid specimens from patients and rats were mainly used for immunofluorescence staining to detect potential target proteins (Figure 1C). We used the NECTIN2 antibody from Proteintech (catalogue number: 27171-1AP; Rosemont, IL, USA), PECAM1 antibody from Abcam (catalogue number: ab64543; Cambridge, UK) and CD68 antibody from Abcam (catalogue number: ab125212;
Cambridge, UK). The protocol about staining is described in Supplementary Methods.

\section{MR study}

All the genetic variants associated with LDL-C were acquired from a large genome-wide association study (GWAS) meta-analysis with 173,082 subjects. ${ }^{[20]}$ For the CA dataset, we obtained the corresponding genetic variants from 150,765 subjects including 7,992,739 SNPs. ${ }^{[21]}$ A two-sample MR study was performed to investigate the causal links between LDL-C and CA and identify key genes (Figure 1A). For the selection of important SNPs, we chose SNP with $P$ value $<5 \times 10^{-8}$. In total, $97 \mathrm{SNPs}$ were identified. MR analysis was conducted in $\mathrm{R}$ (version 4.02) using the two-sample MR package, and details are available in Supplementary Methods.

\section{Deep mining of scRNA-seq and DNA microarray data}

Single-cell sequencing data of carotid plaque representing three $\mathrm{AC}$ and three PA regions in six patients undergoing CEA (Figure 1B) were downloaded from Gene Expression Omnibus (GSE159677). Data analyses were performed in $\mathrm{R}$ (version 4.02), and details are available in Supplementary Methods. Fourteen human carotid plaques were downloaded from the database (GSE28829), including seven early-stage carotid plaques (intimal thickening and intimal xanthoma) and seven advanced-stage carotid plaques (thin or thick fibrous cap atheroma with stenosis greater than 70\%). They were analysed using the 'GEO2R' tool. ${ }^{[22]}$ Volcano plots, box plots and uniform manifold approximation and projection (UMAP) were used to visualise gene expression. Adjusted $P$ values were calculated using the BenjaminiHochberg method, and the significance level cut-off was set at 0.05 .

\section{Statistical analysis}


Li et al.: Lipid-related protein NECTIN2 is an important marker in the progression of carotid atherosclerosis

\begin{tabular}{llll}
\hline Table 2: Main MR results of the effect of LDL-C on carotid atherosclerosis \\
\hline Method & SNP $(n)$ & OR (95\% CI) & $P$ \\
\hline MR-Egger & 97 & $1.37(1.13-1.67)$ & $1.79 \times 10^{-3}$ \\
Weighted median & 97 & $1.24(1.08-1.44)$ & $2.94 \times 10^{-3}$ \\
Inverse variance weighted & 97 & $1.29(1.14-1.46)$ & $0.05 \times 10^{-3}$ \\
Weighted mode & 97 & $1.30(1.13-1.50)$ & $0.42 \times 10^{-3}$ \\
\hline
\end{tabular}

$\mathrm{Cl}$ : confidence interval; MR: Mendelian randomisation; LDL: low density lipoprotein cholesterol; SNP: single-nucleotide polymorphism; OR: odds ratio.

\begin{tabular}{|c|c|c|c|c|c|c|}
\hline \multirow[t]{2}{*}{ Method } & \multicolumn{2}{|c|}{ Heterogeneity test } & \multicolumn{2}{|c|}{ Multiplicative random-effects test } & \multicolumn{2}{|c|}{ MR-Egger test } \\
\hline & $\mathbf{Q}$ & $P$ & OR $(95 \% \mathrm{CI})$ & $P$ & OR $(95 \% \mathrm{CI})$ & $P$ \\
\hline Weighted median & 181.44 & $2.30 \times 10^{-7}$ & $2.94 \times 10^{-4}$ & NA & NA & NA \\
\hline $\begin{array}{l}\text { Inverse variance } \\
\text { weighted }\end{array}$ & 182.63 & $2.40 \times 10^{-7}$ & $\begin{array}{l}1.29(1.14- \\
1.46)\end{array}$ & $4.81 \times 10^{-5}$ & NA & NA \\
\hline MR-Egger analysis & NA & NA & NA & NA & $\begin{array}{l}0.99(0.98- \\
1.01)\end{array}$ & 0.43 \\
\hline
\end{tabular}

Cl: confidence interval; MR: Mendelian randomisation; NA: not applicable.

Clinical data were analysed using SPSS (version 21.0). Statistical tests were performed by independent Student's $t$-test or Mann-Whitney test. Continuous variables were expressed as mean $\pm \mathrm{SD}$ or median, while categorical variables were presented as numbers analysed using the $\chi^{2}$ test or Fisher's exact test. Ordinal regression analysis was used to analyse the associations between some factors. The results of immunofluorescence staining were analysed by Image J software (version 1.52a).

\section{RESULTS}

\section{No correlation between clinical blood lipid indices and CA progression}

A total of 108 patients who underwent CEA were enrolled in the study. Baseline data are shown in Table 1. The degree of CA was assessed by colour ultrasound. ${ }^{[19]}$ We found that most blood lipid indices (excluding total cholesterol) of the patients were at the normal level, and there was no statistical difference between these indices and the degree of carotid plaques. We believed that this result might be related to the widespread use of lipid-lowering and plaque-stabilising drugs such as statins. Traditional lipid indices could not truly reflect CA progression under these conditions. To circumvent such medication effects, we explored other methods to identify the indicators that truly reflected the progression of CA.

\section{MR analysis showed NECTIN2 potentially provided a strong causal link between LDL-C level and $C A$}

MR analysis revealed 97 SNPs that potentially provided strong causal links between LDL-C level and CA (Table 2). Sensitivity analysis indicated strong heterogeneities of the genetic variants for atherosclerosis $\left(P=2.30 \times 10^{-7}\right.$; Table 3). Therefore, a multiplicative random-effects model was used in the analysis of MR effect size (odds ratio $[\mathrm{OR}]=1.29,95 \%$ confidence interval $[\mathrm{C}]=1.14-1.46$, $P=4.81 \times 10^{-5} ;$ Table 3$)$. These analyses showed that there were causal links between LDL-C level and CA. MR-Egger analysis showed no evidence of pleiotropy in SNPs (OR = $0.99,95 \% \mathrm{CI}=0.98-1.01, P=0.43$; Table 3 ), and leaveone-out analysis did not identify any SNPs that might affect the link. However, leaving out the SNP rs7254892 would cause the sensitivity value to decrease (Figure 2A). Therefore, this SNP could significantly increase the risk of CA based on Wald ratio test (MR effect size $>0$; Figure $2 \mathrm{~B})$. This $\mathrm{SNP}$ is an $\mathrm{A} / \mathrm{G}$ single-nucleotide variation in the NECTIN2 gene. ${ }^{[23]}$ Together, we reasoned that NECTIN2, a gene previously assigned a proatherogenic role, might be associated with CA.

\section{NECTIN2 transcription correlated with CA progression based on scRNA-seq and microarray data mining}

After applying quality control filters, 14,812 cells from the $\mathrm{AC}$ region and 10,374 cells from the PA region were included in the scRNA-seq analysis. The gene expression data were aligned and projected in a two-dimensional space through UMAP, helping us identify the different types of cell populations in the two regions (Figure $3 \mathrm{~A}$ and $\mathrm{C}$ ). To group cells with similar gene expressions, we applied unsupervised Seurat-based clustering and detected 21 and 15 distinct cell clusters from the AC and PA regions, respectively. All clusters were singled out with the top two upregulated markers for each cluster (Figure 3B and D). With established canonical cell markers, each cluster has been assigned a unique biological identity, such as 


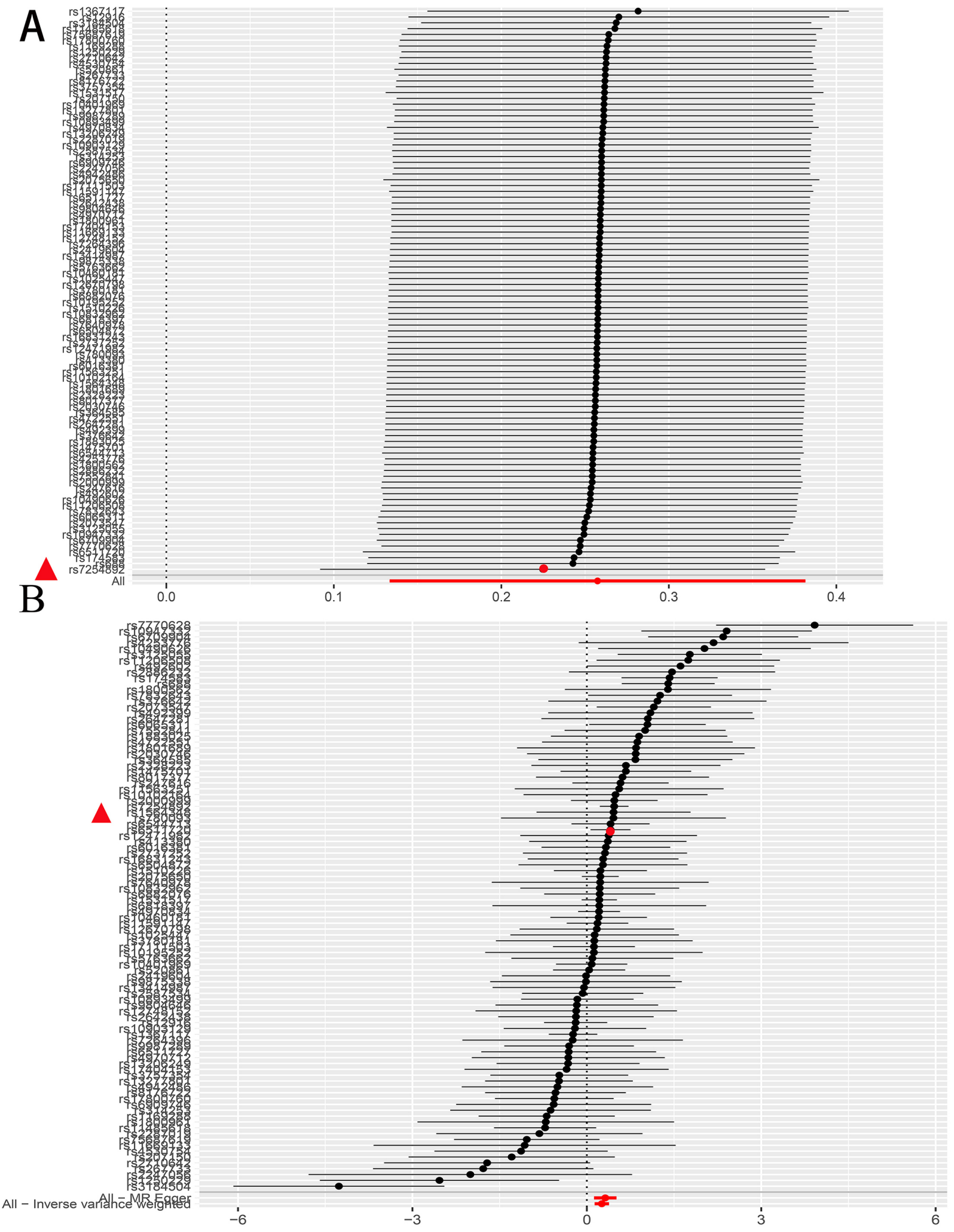

Figure 2: MR study. (A) MR leave-and-out sensitivity analysis. All the lines are on the right side of 0 , which means: (1) the increase of LDL-C could increase the risk of carotid atherosclerosis and (2) the MR result was stable. (B) MR effect size. Each horizontal solid line in the forest map reflects the results estimated by a single SNP: (1) the solid line is completely to the left of 0 , indicating that the result estimated by this SNP was that the increase in LDL-C reduced the risk of carotid atherosclerosis; (2) the solid line is completely to the right of 0 , indicating that the estimated result of this SNP was that LDL-C increased the risk of carotid atherosclerosis and (3) the results that cross zero are not significant (red triangles and dots represent important data, rs7254892). MR: Mendelian randomisation; SNP: single-nucleotide polymorphism; LDL-C: low-density lipoprotein cholesterol. 

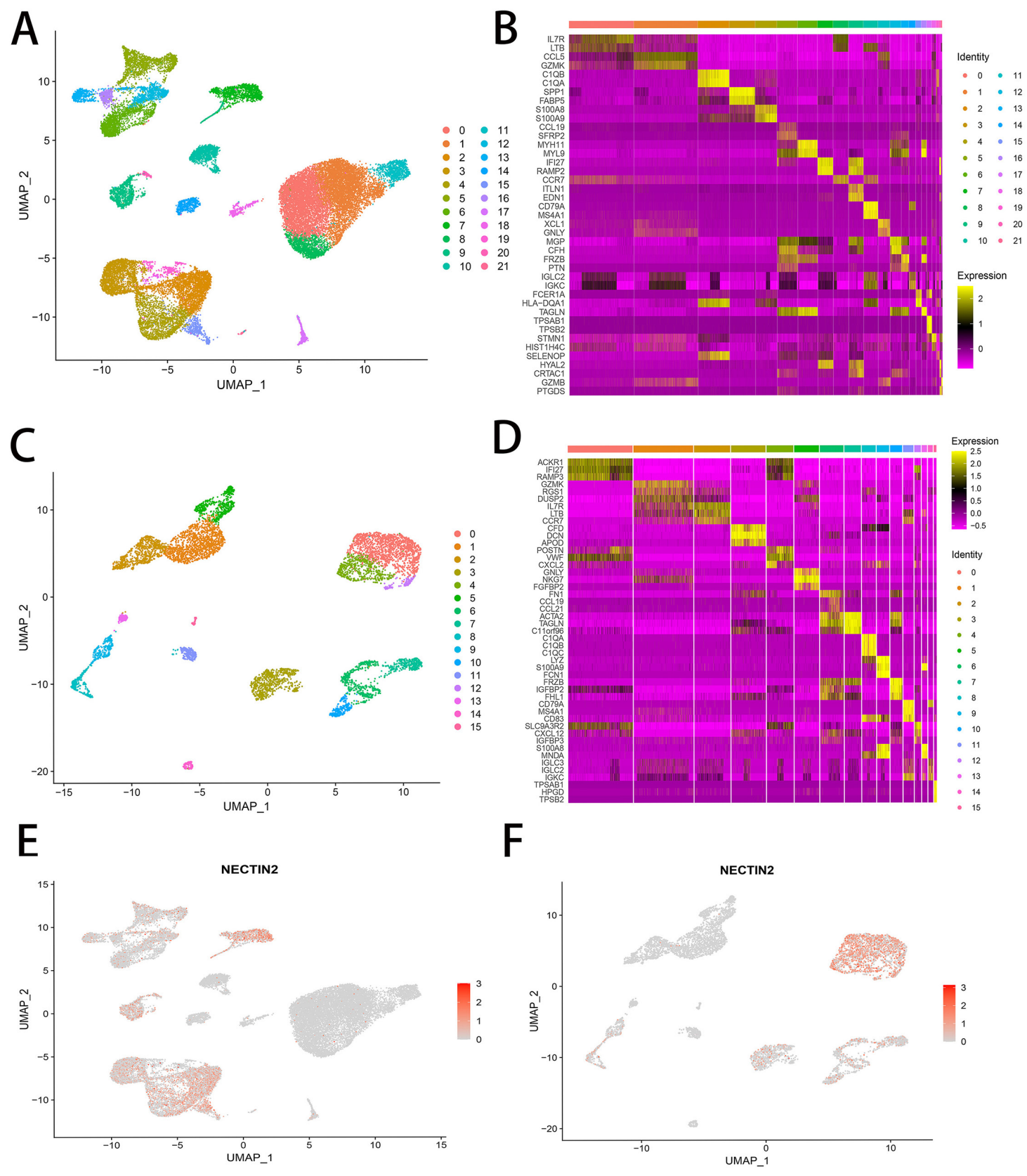

Figure 3: Single-cell RNA sequence data of carotid plaques. (A) UMAP visualisation of cells in AC region. (B) Heatmap showing top two markers among AC cell clusters. (C) UMAP visualisation of cells in PA region. (D) Heatmap showing top two markers among PA cell clusters. (E) Feature plot showing the expression of NECTIN2 in AC region. (F) Feature plot showing the expression of NECTIN2 in PA. AC: atherosclerotic core; PA: proximal adjacent; UMAP: uniform manifold approximation and projection.

endothelial cells (ECs), ${ }^{[24]}$ vascular smooth muscle cells, ${ }^{[25,26]}$ $\mathrm{T}$ cells and macrophages (Supplementary Figures 1-4). ${ }^{[27]}$ Importantly, NECTIN2 was found to be highly expressed in ECs in the AC and PA regions and in macrophages located in the $\mathrm{AC}$ region (Figure $3 \mathrm{E}$ and $\mathrm{F}$ ).

We sought to unravel the phenotypic differences between these endothelial and macrophage populations with a high expression of NECTIN2 in plaques between the AC and PA regions. Our results suggested that NECTIN2 expression was higher in ECs in the PA region than those in the AC region $\left(\log \mathrm{FC}=0.42, P=1.34 \times 10^{-37}\right.$; Figure $\left.4 \mathrm{~A}-\mathrm{C}\right)$. In the PA region, gene ontology (GO) analysis of upregulated genes (including NECTIN2) suggested that multiple functions were enriched, such as regulation of response to biotic stimulus, antigen receptor-mediated signalling pathway and T cell-mediated immunity (Figure 4D). Kyoto Encyclopedia of Genes and Genomes (KEGG) functional enrichment analysis reported signatures, including adhesion molecules and herpes simplex virus 1 infection (HSV-1), 
A

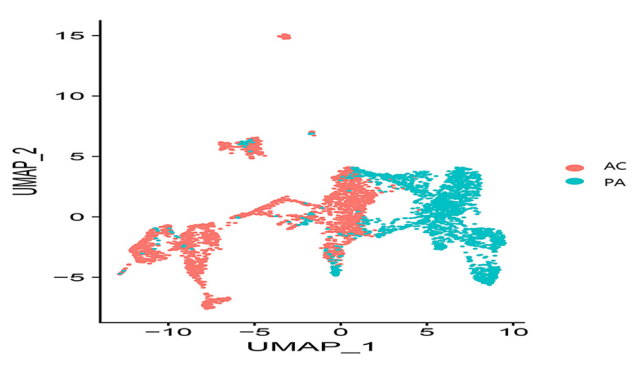

$\mathrm{C}$

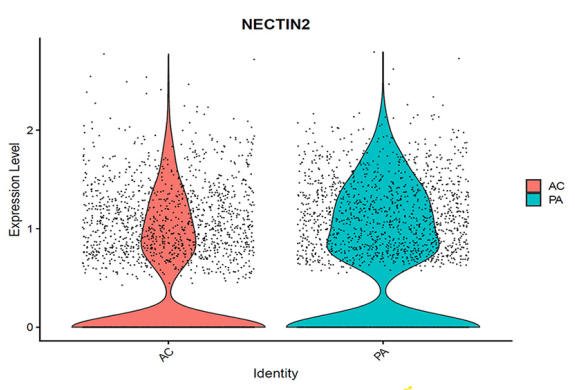

$\mathrm{E}$

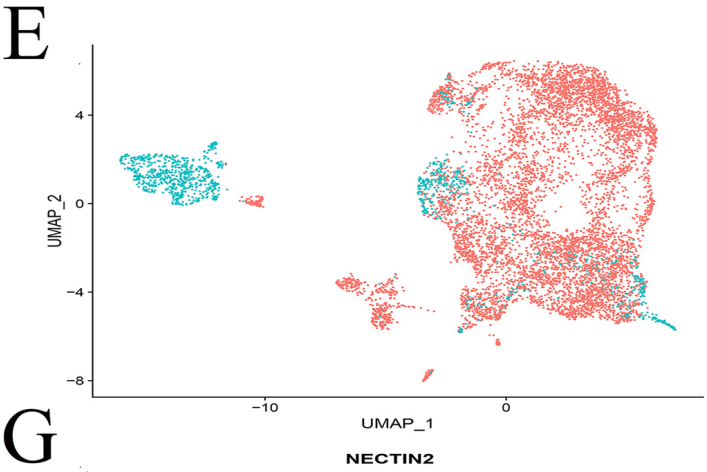

B

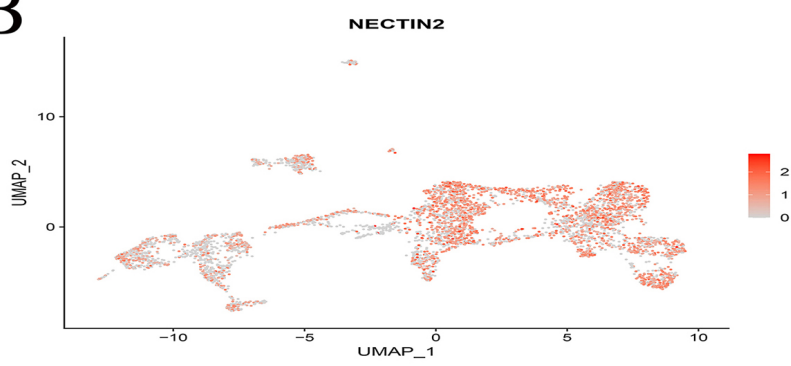

D

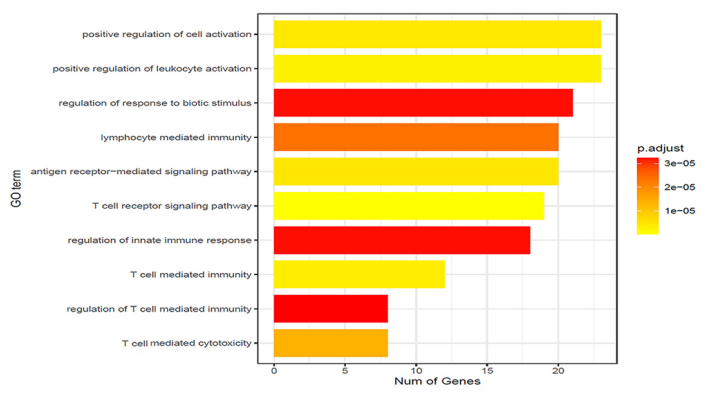

$\mathrm{F}$

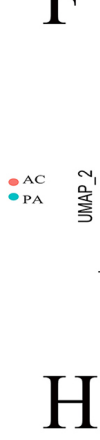

$\boxminus_{\mathrm{PA}}^{\mathrm{AC}}$

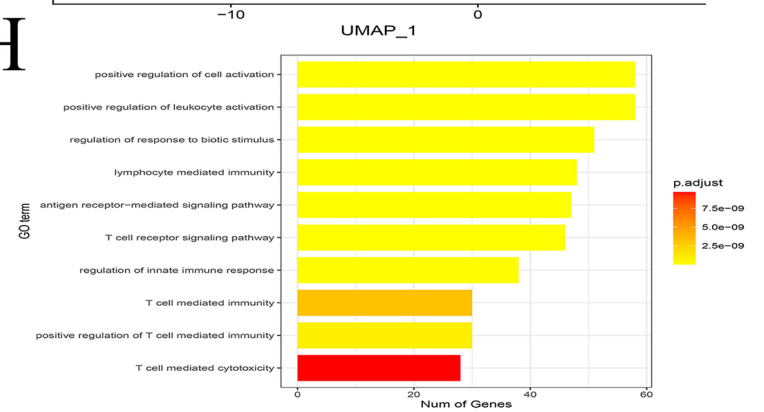

Figure 4: The expression and function of NECTIN2 in endothelial cells and macrophages of carotid plaques. (A) UMAP visualisation of endothelial cells in AC and PA regions. (B) Feature plot showing the expression of NECTIN2 in endothelial cells of the two regions. (C) Violin plot clearly showing the different expression of NECTIN2 in endothelial cells of the two regions. (D) GO analysis of significantly high-expression genes (including NECTIN2) in PA endothelial cells. (E) UMAP visualisation of macrophages in AC and PA regions. (F) Feature plot showing the expression of NECTIN2 in macrophages of the two regions. (G) Violin plot clearly showing the different expression of NECTIN2 in macrophages of the two regions. (H) GO analysis of significantly high-expression genes (including NECTIN2) in AC macrophages. AC: atherosclerotic core; GO: gene ontology; PA: proximal adjacent; UMAP: uniform manifold approximation and projection.

in the database for NECTIN2 (Supplementary Figure 5A and B). The violin plot clearly showed that NECTIN2 expression was significantly higher in macrophage in the $\mathrm{AC}$ region than that in the $\mathrm{PA}$ region $(\log \mathrm{FC}=$ $0.49, P=4.78 \times 10^{-39}$; Figure $\left.4 \mathrm{E}-\mathrm{G}\right)$. GO analysis of upregulated genes (including NECTIN2) suggested that T cell-mediated cytotoxicity and immunity functions were enriched in AC macrophages (Figure 4H). The enriched
KEGG pathway for NECTIN2 in AC macrophages was HSV-1 infection (Supplementary Figure 5B).

To further verify our results, we obtained the data of 14 samples, with seven representing early-stage CA and the remaining seven representing advanced-stage CA (Figure 5). The selected samples were suitable for differential expression analysis because of similar median distribution 

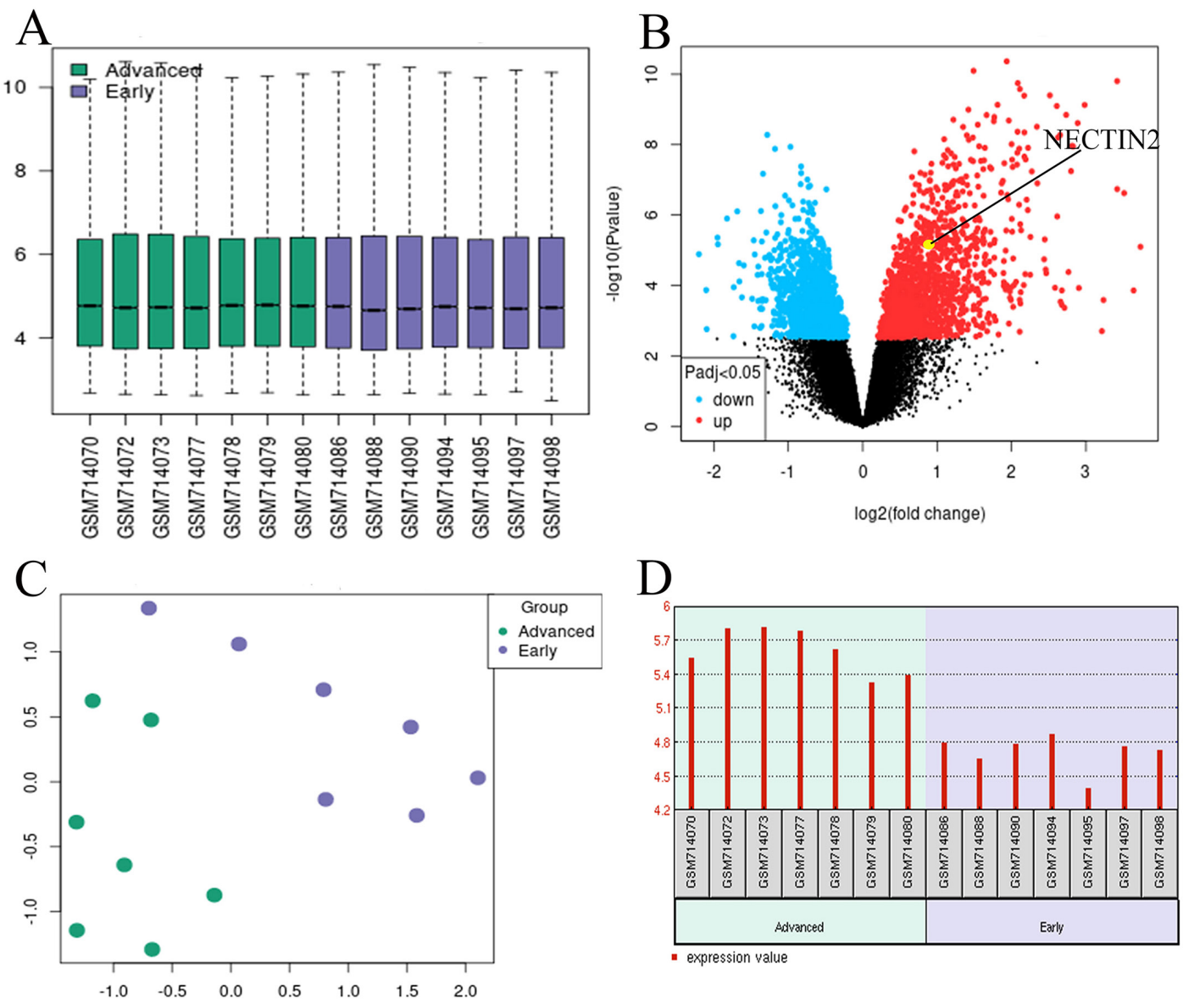

Figure 5: DNA microarray sequence data of early and advanced carotid plaques. (A) Box plot showing similar level in the expression of genes. (B) Volcano plot displaying statistical significance (-log10 $P$ value) versus magnitude of change (log2 fold change), and that NECTIN2 was highly expressed in advanced plaques. (C) UMAP visualisation of early and advanced plaques. (D) Profile graphs of NECTIN2 in early and advanced plaques are shown. UMAP: uniform manifold approximation and projection.

(Figure 5A). The volcano plot displayed a statistical significance $(-\log 10 P$ value) versus the magnitude of change (log2 fold change) and was useful for visualising differentially expressed genes. Through GEO analysis, we found that many genes were differentially expressed between early-stage and advanced-stage CA (Figure 5B). Among them, NECTIN2 was overexpressed in advancedstage CA (Figure 5B). The UMAP plot clearly separated the two types of samples and showed evident differences (Figure 5C). Specifically, the expression of NECTIN2 was significantly upregulated in advanced plaques $(\log \mathrm{FC}=$ $0.90, P=7.04 \times 10^{-5}$; Figure 5D).

Taken together, our results showed that NECTIN2 might be highly expressed in ECs in early-stage CA and in macrophages in advanced-stage CA. Based on enrichment analysis of NECTIN2 functions, we believed that the overexpression of NECTIN2 in ECs in early-stage CA enhanced cell-cell adhesion and increased macrophage adhesion and leukocyte migration at the lesion sites, and that the upregulation of NECTIN2 in macrophages profoundly affected the local lipid metabolism and inflammation in advanced-stage CA.

\section{Verification of NECTIN2 expression changes in CA progression}

The changes of NECTIN2 expression in CA progression were verified using rat and clinical specimens. Through weekly monitoring, we found that the serum levels of LDL-C, triglyceride and total cholesterol in the $\mathrm{APOE}^{-1}$ WD group were significantly higher than those in the ND group (Figure 6A-C). The carotid artery turbulence of $\mathrm{APOE}^{-/}$rats was significantly enhanced, and the carotid intima-media thickness slightly increased $(0.12 \pm 0.02 \mathrm{vs}$. $0.10 \pm 0.01, P=0.031)$ after 11 weeks of WD feeding compared to 5 weeks of WD feeding (Figure 6D-F), suggesting the successful modelling of the early process of $\mathrm{CA}$ in $\mathrm{APOE}^{-/}$rats. Carotid plaques were acquired from CEA (Figure 6G) and detected by carotid colour ultrasound, which showed that clinical CEA specimens 

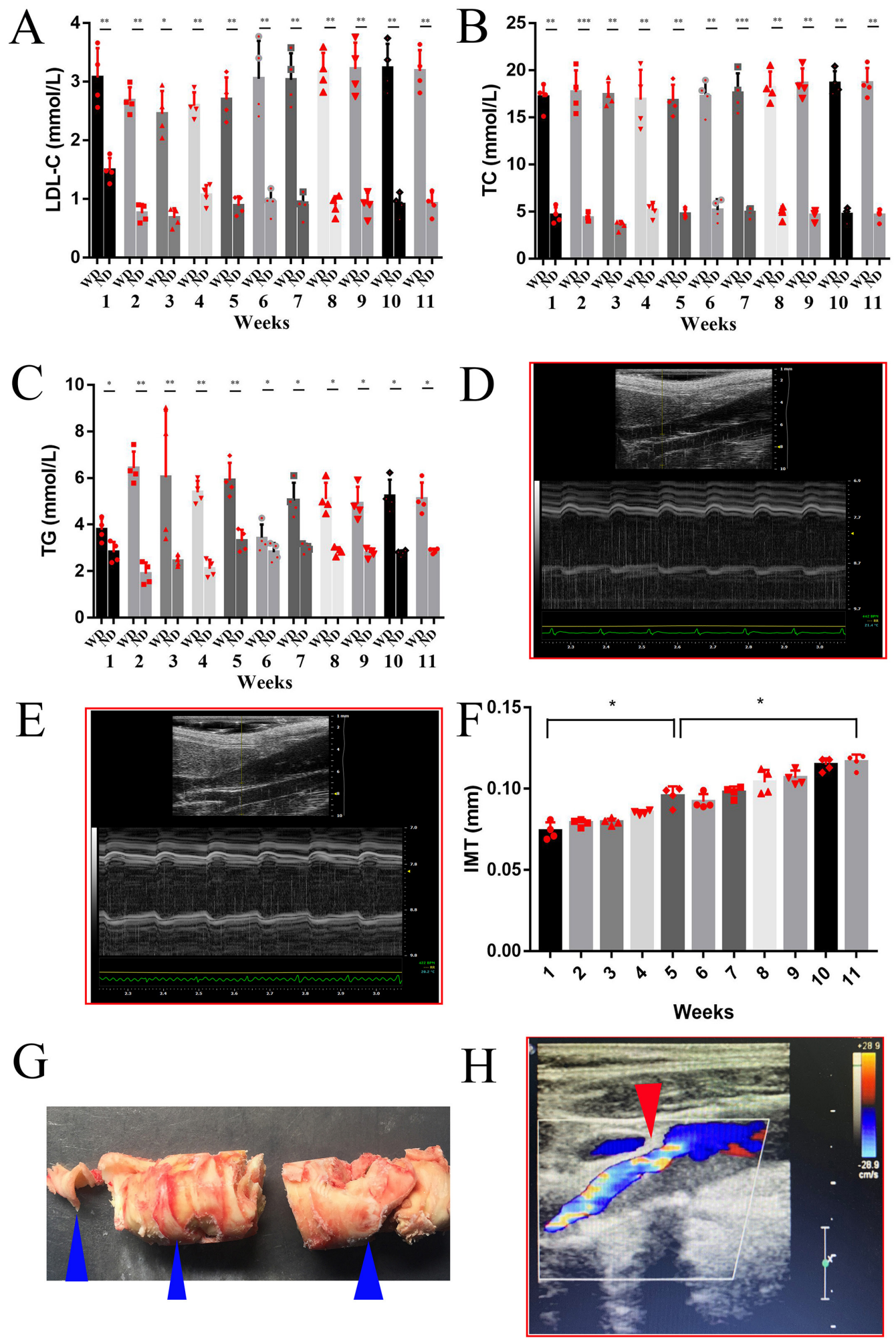

Figure 6: The information about APOE $/$ rats and clinical plaque specimens. (A-C) The levels of LDL-C, TG and TC in WD (n=4) and ND (n=4) groups. (D) The results of carotid artery of $A P O E^{-/}$rats (WD, 5 weeks) obtained by animal colour ultrasound. (E) The results of carotid artery of APOE-/ rats (WDD, 11 weeks) obtained by animal colour ultrasound. (F) The results of carotid IMT of APOE-/ rats (WD, 1-11 weeks, $n=4$ ) obtained by animal colour ultrasound. (G) The clinical specimens of human carotid plaques (blue triangles represent proximal adjacent region, atherosclerotic core region and atherosclerotic core region from left to right). (H) Patients' results (stenosis $>70 \%$ ) obtained by colour ultrasound (red triangle represents stenosis). ${ }^{*} P<0.05,{ }^{* *} P<0.01,{ }^{* * * P}<$ 0.001. IMT: intima-media thickness; LDL-C: low-density lipoprotein cholesterol; ND: normal diet; TC: total cholesterol; TG: triglyceride; WD: western diet. 
were in the advanced stages of CA (Figure 6H). By combining the carotid specimens from $\mathrm{APOE}^{-/-}$rats and $\mathrm{CEA}$, we were able to model the entire progression of $\mathrm{CA}$.

Consistent with the transcription analysis results, the expression of NECTIN2 protein was clearly viewed in $\mathrm{PECAM}^{+}$ECs in the enface staining of $\mathrm{APOE}^{-/} \mathrm{WD}$ rats and was found to be dramatically enhanced as WD feeding time increased $(1.31 \pm 0.24$ vs. $3.98 \pm 0.41$ vs. $9.57 \pm 0.80$, $P=0.003$; Figure $7 \mathrm{~A}$ and D). NECTIN2 was also highly expressed in CEA specimens. NECTIN2 expression was significantly higher in ECs in the PA region (74.52 \pm 7.69 vs. $17.66 \pm 1.86, P=6.34 \times 10^{-4}$; Figure $7 \mathrm{~B}$ and $\left.\mathrm{E}\right)$, but higher in macrophages in the AC region $(78.83 \pm 5.79$ vs. 29.75 $\pm 9.55, P=9.07 \times 10^{-4}$; Figure $7 \mathrm{C}$ and $\left.\mathrm{E}\right)$. Interestingly, we also found that most NECTIN2 ${ }^{+} / \mathrm{CD} 68^{+}$cells were distributed in the fibrous cap of plaques, suggesting that NECTIN2 might not only promote CA progression, but also be involved in plaque stability.

\section{DISCUSSION}

Our major findings were as follows: (1) NECTIN2 might be associated with CA through lipid metabolism and (2) NECTIN2 expression exhibited cell specificity and significantly changed as CA progressed.

NECTIN2 is a cell adhesion molecule that mediates cell adhesion and cell signalling through cis- and transdimerisation. ${ }^{[28,29]}$ Adjacent cells are initially connected by the cis-dimer molecules of NECTIN2, whose trans-dimer molecules activate intracellular protein kinase B and Cdc42, and then microfilaments are rearranged through the IQGAP1 protein, recruiting cadherin to participate in the cell junction. ${ }^{[2]}$ NECTIN2 is also involved in mediating the entry of viruses, such as certain mutant strains of HSV and pseudorabies virus, into cells. ${ }^{[30,31]}$ NECTIN2 also contributes to tumour immunity. ${ }^{[32,33]}$ Studies have shown that NECTIN2 can act as a ligand for CD226 receptors in nature killer cells, transmit activated signals to cells, promote the killing of tumour cells and participate in innate and adaptive immune responses. ${ }^{[2]}$ However, a few studies have focused on the relationship between NECTIN2 and atherosclerosis. One MR study suggested that NECTIN2 likely affected the occurrence of lipid-induced atherosclerosis, ${ }^{[11]}$ while another study showed that NECTIN2-deficient mice had significantly fewer atherosclerotic lesions and more stable plaques than normal mice. ${ }^{[8]}$ However, key issues, such as changes in NECTIN2 expression and its pathological functions in the progression of atherosclerosis, have not been addressed.

Consistent with the results of some studies, ${ }^{[34]}$ our results showed that traditional blood lipid indices, such as highdensity lipoprotein cholesterol (HDL-C) and LDL-C, could not explain the progression of CA. However, the high lipid level, especially LDL-C level, is one of the main risk factors for atherosclerosis. Thus, we used the MR method to identify the casual link between LDL-C and CA and found potential important genes, including NECTIN2. Therefore, we hypothesised that the progression of atherosclerosis could not be elucidated using traditional targets, but could be evaluated using the expression of key genes. We then analysed the single-cell data of carotid plaques. After matching the MR results, we found that only NECTIN2 was significant. In carotid plaques, the expression of NECTIN2 was increased in ECs in earlystage $C A$ and in macrophages in advanced-stage CA. In addition, we further confirmed that NECTIN2 expression was related to CA progression using microarray data of early-stage and advanced-stage carotid plaques. Finally, we used human CEA and $\mathrm{APOE}^{-/}$rat specimens to model the entire progression of $\mathrm{CA}$ and revealed the specific expression changes of NECTIN2 in various cell types from early to advanced stage.

Regarding the function of NECTIN2 protein in CA, we focused on the 'bridge function' of NECTIN2 and CD226 in our protein-protein interaction network analysis (Supplementary Figure 6A). ${ }^{[3]}$ We found that lipid proteins and inflammatory proteins were linked by the ligand-receptor binding of NECTIN2 and CD226. ${ }^{[36]}$ As we all know, lipid metabolism plays an important role in CA progression. Thus, we thought that lipid-related protein NECTIN2 might act as a mediator to immune response in the process of dyslipidaemia in CA. Then, we found that $\mathrm{T}$ cells were the only cell type expressing CD226 in plaques (Supplementary Figures 3 and 6B, C), which should proliferate and upregulate CD226 expression if activated by NECTIN2. ${ }^{[37]}$ Although the subtypes of T cells in two regions were different (Supplementary Figure 6D), as the receptor of NECTIN2, CD266 expression did not show significant differences on $\mathrm{T}$ cells between the $\mathrm{PA}$ and $\mathrm{AC}$ regions $(\log \mathrm{FC}=0.12, P=0.891$; Supplementary Figure 6E). Therefore, we inferred that NECTIN2 is mainly involved in intercellular adhesion rather than cell signalling by ligand-receptor binding in the progression of CA. In addition, since NECTIN2 was highly expressed in the fibrous cap area of carotid plaques (Figure 7B and C), we speculated that it might be related to plaque stability. ${ }^{[8,38]}$

NECTIN2 also acts as a receptor for HSV-1. Previous studies have indicated that HSV-1 infection could increase the risk of atherosclerosis. ${ }^{[39,40]}$ However, no research has illustrated the role of NECTIN2 in this relationship. Our results, which provided a better understanding of the pathological process of atherosclerosis, can elucidate the potential mechanism between HSV-1 infection and its 

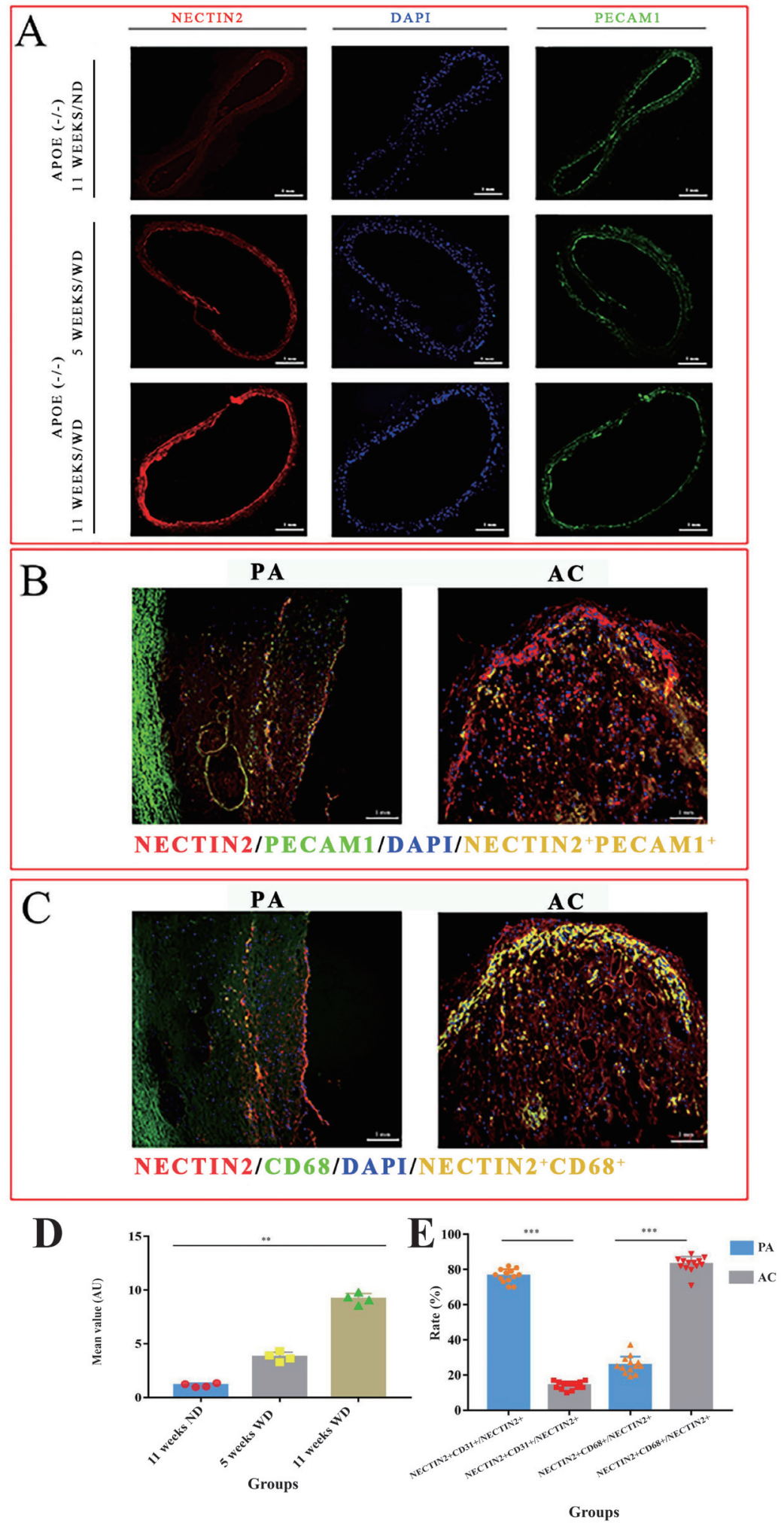

Figure 7: The enface staining of APOE $/$ rats and clinical plaque specimens. (A and D) The mean value of NECTIN2 was higher in APOE ${ }^{-/}$rats (western diet, 11 weeks, $n=4$ ) (NECTIN2 [red], endothelial cells [PECAM1, green] and nucleus [DAPI, blue]) Scale bar: $1 \mathrm{~mm}$. (B-D) NECTIN2 expression was significantly higher in macrophages in the AC region $(n=12)$, but higher in endothelial cells in the PA region $(n=12)$ (NECTIN2 [red], endothelial cells [PECAM1, green], macrophage [CD68, green] and nucleus [DAPI, blue]). Scale bar: $1 \mathrm{~mm} .{ }^{* *} P<0.01,{ }^{* * *} P<0.001$. AC: atherosclerotic core; ND: normal diet; PA: proximal adjacent; WD: western diet. 
associated high incidence of atherosclerosis.

Our study illustrated for the first time that the lipidrelated protein NECTIN2 is related to the progression of CA. Thus, NECTIN2 is an important marker in CA progression and has a potential in becoming a new therapeutic target for clinical prevention. Using big data analysis, we also summarised the differential expression of NECTIN2 in different cell types. However, our study was a single-centre study and selection bias was unavoidable. Therefore, additional well-designed and larger clinical trials are required in the future.

\section{Source of Funding}

Ethics approval and consent to participate

Our study was approved by the Ethics Committee of the First Affiliated Hospital of Zhengzhou University (2020KY-0067-001), and all animal studies were approved by the Ethics Committee of the Institutional Animal Care and Use Committee at Zhengzhou University (2020-zzuIACUC-0172).

This work was supported by the National Natural Science Foundation of China grant number 31970158 to ZX and 81530037 to YX.

\section{Conflict of Interests}

None declared.

\section{REFERENCES}

1. Libby P, Buring JE, Badimon L, Hansson GK, Deanfield J, Bittencourt MS, et al. Atherosclerosis. Nat Rev Dis Primers 2019;5:56.

2. Song P, Fang Z, Wang H, Cai Y, Rahimi K, Zhu Y, et al. Global and regional prevalence, burden, and risk factors for carotid atherosclerosis: A systematic review, meta-analysis, and modelling study. Lancet Global Health 2020;8:e721-9.

3. Urban D, Poss J, Bohm M, Laufs U. Targeting the proprotein convertase subtilisin/kexin type 9 for the treatment of dyslipidemia and atherosclerosis. J Am Coll Cardiol 2013;62:1401-8.

4. Ntaios G, Wintermark M, Michel P. Supracardiac atherosclerosis in embolic stroke of undetermined source: The underestimated source. Eur Heart J 2021;42:1789-96.

5. Hoogeveen RM, Nahrendorf M, Riksen NP, Netea MG, de Winther MPJ, Lutgens $\mathrm{E}$, et al. Monocyte and haematopoietic progenitor reprogramming as common mechanism underlying chronic inflammatory and cardiovascular diseases. Eur Heart J 2018;39:3521-7.

6. Mistriotis P, Andreadis ST. Vascular aging: Molecular mechanisms and potential treatments for vascular rejuvenation. Ageing Res Rev 2017;37:94-116.

7. Jenkins AJ, Welsh P, Petrie JR. Metformin, lipids and atherosclerosis prevention. Curr Opin Lipidol 2018;29:346-53.

8. Rossignoli A, Shang MM, Gladh H, Moessinger C, Foroughi Asl H, Talukdar HA, et al. Poliovirus receptor-related 2: A cholesterol-responsive gene affecting atherosclerosis development by modulating leukocyte migration. Arterioscler Thromb Vasc Biol 2017;37:534-42.

9. Blaschuk OW, Rowlands TM. Plasma membrane components of adherens junctions (review). Mol Membr Biol 2002;19:75-80.

10. Nakanishi H, Takai Y. Roles of nectins in cell adhesion, migration and polarization. Biol Chem. 2004;385:885-92.

11. van der Graaf A, Claringbould A, Rimbert A, Consortium B, Westra HJ, $\mathrm{Li} \mathrm{Y}$, et al. Mendelian randomization while jointly modeling cis genetics identifies causal relationships between gene expression and lipids. Nat Commun 2020;11:4930.

12. Lichtman JH, Jones MR, Leifheit EC, Sheffet AJ, Howard G, Lal BK, et al. Carotid endarterectomy and carotid artery stenting in the us medicare population, 1999-2014. JAMA 2017;318:1035-46.

13. Knappich C, Kuehnl A, Haller B, Salvermoser M, Algra A, Becquemin JP, et al. Associations of perioperative variables with the 30-day risk of stroke or death in carotid endarterectomy for symptomatic carotid stenosis. Stroke 2019;50:3439-48.

14. Berenji Ardestani S, Eftedal I, Pedersen M, Jeppesen PB, Norregaard R, Matchkov VV. Endothelial dysfunction in small arteries and early signs of atherosclerosis in apoe knockout rats. Sci Rep 2020;10:15296.

15. Latvala A, Ollikainen M. Mendelian randomization in (epi)genetic epidemiology: An effective tool to be handled with care. Genome Biol 2016;17:156.

16. Hoheisel JD. Microarray technology: Beyond transcript profiling and genotype analysis. Nat Rev Genet 2006;7:200-10.

17. Wu Y, Zhang $\mathrm{K}$. Tools for the analysis of high-dimensional single-cell rna sequencing data. Nat Rev Nephrol 2020;16:408-21.

18. Tang F, Barbacioru C, Wang Y, Nordman E, Lee C, Xu N, et al. Mrna-seq whole-transcriptome analysis of a single cell. Nat Methods 2009;6:377-82.

19. Wangqin R, Krafft PR, Piper K, Kumar J, Xu K, Mokin M, et al. Management of de novo carotid stenosis and postintervention restenosiscarotid endarterectomy versus carotid artery stenting-a review of literature. Transl Stroke Res 2019;10:460-74.

20. Willer CJ, Schmidt EM, Sengupta S, Peloso GM, Gustafsson S, Kanoni $\mathrm{S}$, et al. Discovery and refinement of loci associated with lipid levels. Nat Genet 2013;45:1274-83.

21. Malik R, Chauhan G, Traylor M, Sargurupremraj M, Okada Y, Mishra A, et al. Multiancestry genome-wide association study of 520,000 subjects identifies 32 loci associated with stroke and stroke subtypes. Nat Genet 2018;50:524-37.

22. Dumas J, Gargano MA, Dancik GM. Shinygeo: A web-based application for analyzing gene expression omnibus datasets. Bioinformatics 2016;32:3679-81.

23. Lin R, Zhang Y, Yan D, Liao X, Gong G, Hu J, et al. Association of common variants in tomm40/apoe/apoc1 region with human longevity in a chinese population. J Hum Genet 2016;61:323-8.

24. Kalucka J, de Rooij L, Goveia J, Rohlenova K, Dumas SJ, Meta E, et al. Single-cell transcriptome atlas of murine endothelial cells. Cell 2020;180:764-9.e720.

25. Li Z, Solomonidis EG, Meloni M, Taylor RS, Duffin R, Dobie R, et al. Single-cell transcriptome analyses reveal novel targets modulating cardiac neovascularization by resident endothelial cells following myocardial infarction. Eur Heart J 2019;40:2507-20.

26. Rohlenova K, Goveia J, Garcia-Caballero M, Subramanian A, Kalucka J, Treps L, et al. Single-cell rna sequencing maps endothelial metabolic plasticity in pathological angiogenesis. Cell Metab 2020;31:862-77.e814.

27. Fernandez DM, Rahman AH, Fernandez NF, Chudnovskiy A, Amir ED, Amadori L, et al. Single-cell immune landscape of human atherosclerotic plaques. Nat Med 2019;25:1576-88.

28. Mandai K, Rikitake Y, Mori M, Takai Y. Nectins and nectin-like molecules in development and disease. Curr Top Dev Biol 2015;112:197-231.

29. Molfetta R, Milito ND, Zitti B, Lecce M, Fionda C, Cippitelli M, et al. The ubiquitin-proteasome pathway regulates nectin $2 / \mathrm{cd} 112$ expression and impairs nk cell recognition and killing. Eur J Immunol 2019;49:873-83. 
30. Martinez WM, Spear PG. Structural features of nectin-2 (hveb) required for herpes simplex virus entry. J Virol 2001;75:11185-95.

31. Ho DW, Tsui YM, Chan LK, Sze KM, Zhang X, Cheu JW, et al. Singlecell rna sequencing shows the immunosuppressive landscape and tumor heterogeneity of hbv-associated hepatocellular carcinoma. Nat Commun 2021;12:3684.

32. Papanicolau-Sengos A, Yang Y, Pabla S, Lenzo FL, Kato S, Kurzrock R, et al. Identification of targets for prostate cancer immunotherapy. Prostate 2019;79:498-505.

33. Wang JB, Li P, Liu XL, Zheng QL, Ma YB, Zhao YJ, et al. An immune checkpoint score system for prognostic evaluation and adjuvant chemotherapy selection in gastric cancer. Nat Commun 2020;11:6352.

34. Fernandez-Friera L, Fuster V, Lopez-Melgar B, Oliva B, Garcia-Ruiz JM, Mendiguren J, et al. Normal ldl-cholesterol levels are associated with subclinical atherosclerosis in the absence of risk factors. J Am Coll Cardiol 2017;70:2979-91.

35. Khorsand B, Savadi A, Naghibzadeh M. Comprehensive host-pathogen protein-protein interaction network analysis. BMC Bioinformatics
2020;21:400.

36. Battella S, Oliva S, Franchitti L, La Scaleia R, Soriani A, Isoldi S, et al. Fine tuning of the dnam-1/tigit/ligand axis in mucosal t cells and its dysregulation in pediatric inflammatory bowel diseases (ibd). Mucosal Immunol 2019;12:1358-69.

37. Sinha S, Miller LM, Subramanian S, Burrows GG, Vandenbark AA, Offner H. Rt1551 treatment of eae reduces cd226 and t-bet $+\mathrm{cd} 4 \mathrm{t}$ cells in periphery and prevents infiltration of t-bet+ il-17, ifn-gamma producing $\mathrm{t}$ cells into cns. PLoS One 2011;6:e21868.

38. Bentzon JF, Otsuka F, Virmani R, Falk E. Mechanisms of plaque formation and rupture. Circ Res 2014;114:1852-66.

39. Wu YP, Sun DD, Wang Y, Liu W, Yang J. Herpes simplex virus type 1 and type 2 infection increases atherosclerosis risk: Evidence based on a meta-analysis. Biomed Res Int. 2016;2016:2630865.

40. Alber DG, Vallance P, Powell KL. Enhanced atherogenesis is not an obligatory response to systemic herpesvirus infection in the apoedeficient mouse: Comparison of murine gamma-herpesvirus-68 and herpes simplex virus-1. Arterioscler Thromb Vasc Biol 2018;22:793-8.
How to cite this article: Li S, Gao Y, Ma K, Li Y, liu CH, Yan Y, et al. Lipid-related protein NECTIN2 is an important marker in the progression of carotid atherosclerosis: An intersection of clinical and basic studies. J TransI Intern Med 2021; 9: 294-306. 